MODELING, IDENTIFICATION AND CONTROL, 1995, vOL. 16, NO. 3, 169-176

doi:10.4173/mic.1995.3.5

\title{
Flow over complex terrain estimated by a general purpose Navier-Stokes solver
}

\author{
L. K. ALM $\dagger$ and T. A. NYGAARD $\dagger$
}

Keywords: Wind power, terrain models, Navier-Stokes solver

\begin{abstract}
A general purpose Navier-Stokes solver has been applied to atmospheric flow on microscale. For flat terrain the model produces the well known logarithmic profile in the surface layer and describes the effect of roughness change. At Askervein Hill, good agreement between model results and measurements is observed also behind the hilltop, where linearized models overpredict the wind speed.
\end{abstract}

\section{Introduction}

To get some practical experience with installation and operation of wind turbines, ten wind turbines have been erected in Norway. All are sited along the coast in the western and northern part of the country, which is the most interesting part for wind energy. The terrain varies from small smooth islands leaving the wind nearly undisturbed, to areas with steep mountains some kilometres away from the site, separating the flow. To estimate the expected energy capture, simultaneous wind monitoring at the sites and weather stations with known long term wind data have been used (Nygaard 1992).

The model WASP developed by RIS $\emptyset$ has also been applied. Through comparisons with the measurements, we have become increasingly aware of the limitations of linearized flow model like WASP.

If the use of wind monitoring could be reduced, the savings would be significant. This fact, and the exciting development of computer capacity/price has inspired us to take more of the complex physics of atmospheric flow into account when estimating local terrain effects.

The software package PHOENICS developed by Cham Ltd has been used at Institutt for energiteknikk for some years, mainly for gas flow in tubes. It was therefore possible to test this package for atmospheric flow with moderate effort, as a starting point for development of a siting tool for Norwegian conditions.

\section{Model description}

PHOENICS is a general Navier-Stokes simulation package which can solve the balance equations for mass, momentum, turbulence quantities, energy and chemical species (Spalding 1991).

Several assumptions and simplifications are to be done for adoption to atmospheric flow on the microscale. Since we are mainly interested in low mach-number flow some $0-100 \mathrm{~m}$ above ground, density variations are modest, and a constant density is assumed

Received 6 June 1994

$\dagger$ Institutt for energiteknikk, P. O. Box 40, N-2007 Kjeller, Norway. 
in the simulations. Thereby the momentum equations are decoupled from the energy equation. The energy equation is therefore ignored. The Coriolis force has a modest impact at low heights above ground, and is also neglected. Shear stress, acceleration and pressure gradient terms are dominating. Assuming a neutral atmosphere, gravity is cancelled by buoyancy, and the pressure variable can be defined as the perturbation from the hydrostatic pressure.

The turbulence is modeled by the widely used $k-\varepsilon$ model. $k$ is a symbol for turbulent kinetic energy while $\varepsilon$ is the dissipation rate of $k$. In the $k-\varepsilon$ model the mixing is assumed to be isotropic, i.e. equal in all directions. The model consists of transport equations of $k$ and $\varepsilon$. Thereby the effects of a change in roughness or orography are advected downstream and diffused throughout the domain. This effect cannot be represented by a simple mixing length model, which assumes local equilibrium. In the $k-\varepsilon$ model local equilibrium between production and dissipation of turbulent kinetic energy is assumed only close to the wall (ground), where so-called wall-functions are applied. These functions provide empirical information about the flow regime in the log-law region. The flow conditions at the ground are decided through an iteration that takes into account both the wall functions and the flow immediately above.

\subsection{Equations}

The assumptions above justify the following mathematical simplification. The mass balance is given by

$$
\frac{\partial \rho}{\partial t}+\frac{\partial}{\partial x_{i}}\left(\rho u_{i}\right)=0
$$

where $\rho$ is the density and $u_{1}, u_{2}, u_{3}$ are the time-averaged velocity components. When the density is constant, the equation simply states that the divergence of the velocity vector vanishes.

The time averaging of the Navier-Stokes equations gives rise to the Reynolds stress tensor $\overline{\hat{u}_{i} \hat{u}_{j}}$, where $\hat{u}_{i}$ is the velocity perturbation and $\bar{*}$ means time averaging. The Reynolds stresses $\hat{\hat{u}}_{i} \hat{u}_{j}$ are modeled by using a so-called turbulent viscosity $v_{T}$

$$
-\overline{\hat{u}_{i} \hat{u}_{j}}=2 v_{T}\left(\frac{\partial u_{i}}{\partial x_{j}}+\frac{\partial u_{j}}{\partial x_{i}}\right)-\frac{2}{3} k \delta_{i, j}
$$

which leads to the following equations for the time averaged momentums $u_{i}$

$$
\frac{\partial u_{i}}{\partial t}+u_{j} \frac{\partial u_{j}}{\partial x_{j}}-\frac{\partial}{\partial x_{j}}\left(\left(v_{T}+v_{l}\right) \frac{\partial u_{i}}{\partial x_{j}}\right)+\frac{1}{\rho} \frac{\partial p}{\partial x_{i}}=0
$$

$p$ is the sum of the static pressure perturbation (normal stress) and the turbulent normal stress, equivalent to $\frac{2}{3} p k$. (The molecular viscosity, $v_{l}$, can be neglected because it is significant only some $\mathrm{mm}$ above the ground.)

The turbulent viscosity is defined as the ratio

$$
v_{T}=C_{\mu^{\prime}} k^{2} / \varepsilon
$$

Separate transport equations for $k$ and $\varepsilon$ read as

$$
\frac{\partial k}{\partial t}+u_{j} \frac{\partial k}{\partial x_{j}}-\frac{\partial}{\partial x_{j}}\left(\frac{v_{l}+v_{T}}{\sigma_{k}} \frac{\partial k}{\partial x_{j}}\right)=v_{T}\left(\frac{\partial u_{i}}{\partial x_{j}}+\frac{\partial u_{j}}{\partial x_{i}}\right) \cdot \frac{\partial u_{i}}{\partial x_{j}}-\varepsilon
$$




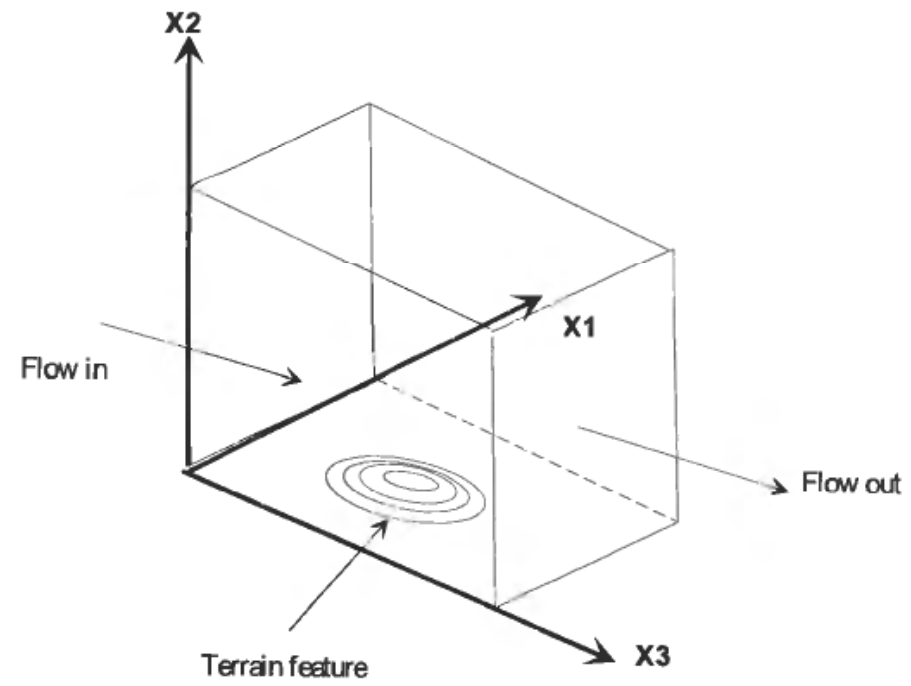

Figure 1. Computational domain.

$$
\frac{\partial \varepsilon}{\partial t}+u_{j} \frac{\partial \varepsilon}{\partial x_{j}}-\frac{\partial}{\partial x_{j}}\left(\frac{v_{l}+v_{T}}{\sigma_{\varepsilon}} \frac{\partial \varepsilon}{\partial x_{j}}\right)=\frac{\varepsilon}{k}\left(C_{\varepsilon 1} P_{k}-C_{\varepsilon 2} \varepsilon\right)
$$

$\sigma_{k}$ and $\sigma_{\varepsilon}$ are the turbulent Prandtl numbers for $k$ and $\varepsilon$ respectively.

The production $P_{k}$ is given by

$$
P_{k}=v_{T}\left(\frac{\partial u_{i}}{\partial x_{j}}+\frac{\partial u_{j}}{\partial x_{i}}\right) \frac{\partial u_{i}}{\partial x_{j}}
$$

while the constants in the $k-\varepsilon$ closure is set to

$$
\begin{aligned}
C_{\mu^{\prime}} & =C_{\mu} \cdot C_{D}=0.0324 \\
\sigma_{k} & =1.0 \quad \sigma_{\varepsilon}=1.85 \\
C_{\varepsilon 1} & =1.44 \quad C_{\varepsilon 2}=1.92
\end{aligned}
$$

This presentation follows Anderson (1988), except for the choice of the numeric value of $C_{\mu^{\prime}}$, where Panofsky and Dutton's (1984) value for $\left(C_{\mu^{\prime}}\right)^{1 / 2}$ for atmospheric flow is selected. To account for this the turbulent Prandtl number for $\varepsilon$ is increased to 1.85 , according to the following equation (Beljaars et al., 1986)

$$
C_{\varepsilon 2}-C_{\varepsilon 1}=\kappa^{2} /\left(\left(C_{\mu^{\prime}}\right)^{1 / 2} \cdot \sigma_{\varepsilon}\right)
$$

$\kappa$ is von Karman's constant, usually assumed to be 0.4 .

The $x_{1}$ and $x_{3}$ axes are oriented horizontally, with $x_{3}$ along the main flow direction $x_{2}$ is oriented vertically. The domain is indicated in figure 1 .

\subsection{Boundary conditions}

The boundary conditions at the ground is determined by empirical laws of the wall, prescribing a logarithmic velocity profile. Denote the velocity parallel to the wall by $u$ and let $y$ be the vertical co-ordinate. Then the boundary condition at the first node 
above ground can be expressed by

$$
u=\frac{u *}{\kappa} \ln \left(E y u * / v_{l}\right)
$$

Let $\tau_{w}$ be the wall shear stress. The friction velocity $u_{*}$, and a skin friction factor $s$ can be defined by

$$
\tau_{w}=\rho u_{*}^{2}=\rho s u^{2}
$$

Combining (9) and (10) and defining $\operatorname{Re}=u y / v_{l}, s$ can be calculated by iterating on

$$
s=\left[\frac{\kappa}{\ln \left(\operatorname{Re} \cdot E \cdot s^{1 / 2}\right)}\right]^{2}
$$

$E$ is set to

$$
\frac{29 \cdot 7 v_{L}}{h_{r} \cdot u_{*}} \text { for } \frac{u_{*} \cdot h_{r}}{v_{L}}>100
$$

If the physical roughness height $h_{r}$ is set to $29.7 y_{0}$, where $y_{0}$ is the aerodynamic roughness, we recognise the usual logarithmic law for the Prandtl layer.

Let $d$ be the distance from the wall to the lowest node. Assuming that production equals dissipation, the boundary conditions for $k$ and $\varepsilon$ near the ground are given by

$$
\begin{aligned}
k & =\frac{u_{*}^{2}}{\left(C_{\mu^{\prime}}\right)^{1 / 2}} \\
\varepsilon & =\frac{u_{*}^{3}}{\kappa d}
\end{aligned}
$$

The flow field entering the domain is chosen to be a fully developed boundary layer. The profile is constructed by running PHOENICS in parabolic mode over a flat plain.

At the outlet zero gradient boundary conditions are imposed. This means that there is no diffusion out of the domain of momentum, dissipation or turbulence, the flow leaving the domain is pure advection. This is not a limitation because the outlet should not be placed in a region with gradients. When $x_{3}$ is the outlet direction, the outlet conditions read

$$
\frac{\partial u_{1}}{\partial x_{3}}=\frac{\partial u_{2}}{\partial x_{3}}=\frac{\partial u_{3}}{\partial x_{3}}=\frac{\partial k}{\partial x_{3}}=\frac{\partial \varepsilon}{\partial x_{3}}=0
$$

At the outlet, the pressure perturbation is set to zero, as a matter of convenience.

$$
p=0
$$

At the top of the boundary layer, and at the lateral boundaries parallel to the inflow, there are imposed symmetry conditions. This means that the velocity components, $u_{n}$, and their derivatives normal to the boundaries are zero.

$$
\frac{\partial u_{n}}{\partial x_{n}}=u_{n}=0
$$

\subsection{Discretisation}

The physical domain is divided into computational cells. The velocity components are represented at the cell interfaces, while the other dependent variables are represented at the cell centres (staggered grid). 
The differential equations are integrated over the control volumes (cells) to form a set of finite difference equations. A so-called hybrid scheme is applied to the convective terms, in order to minimize false diffusion and thereby numerical errors. The finite difference equations are solved by an iterative solving technique: Semi Implicit Pressure Linked Equations (SIMPLE), (Patankar 1980). The grid spacing is implemented to increase upwards with an exponential rate while the grid is uniform in the horizontal directions.

\section{Simulation results}

\subsection{Roughness change on a flat plain}

The roughness change simulations were carried out to test whether the model could reproduce a wind profile over a plain with varying aerodynamic roughness. The boundary conditions were chosen as described above, except for the conditions at the ground, where the aerodynamic roughness was set to $0.01 \mathrm{~m}$ at the inlet, and abruptly changed to $0 \cdot 1 \mathrm{~m}$ one $\mathrm{km}$ downwind. Figure 2 shows the velocity for different heights. The velocity located at $800 \mathrm{~m}, 1100 \mathrm{~m}$ and $1700 \mathrm{~m}$ are shown in fig. 3 .

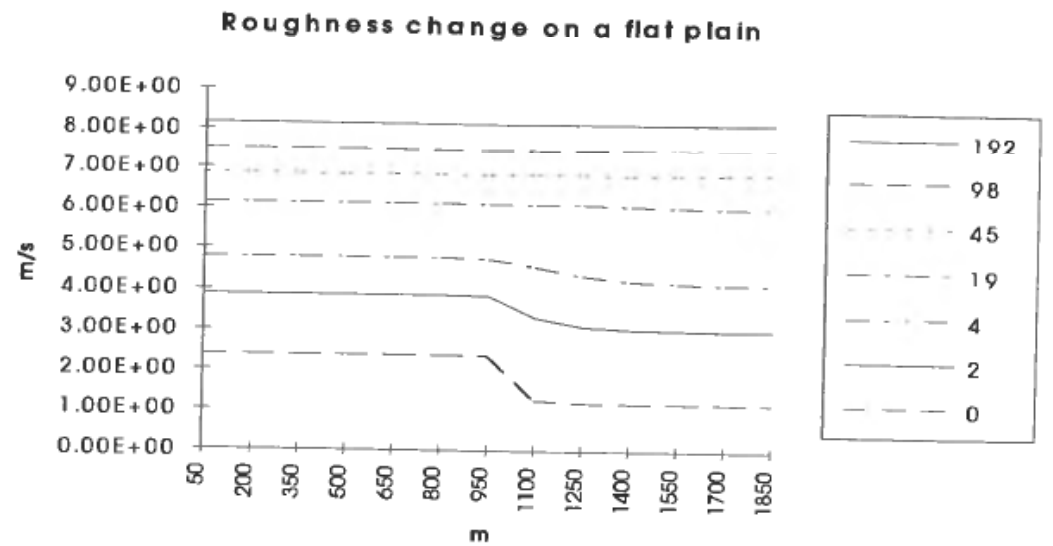

Figure 2. Horizontal velocity at different heights. The horizontal distance is $2000 \mathrm{~m}$, and the roughness change is positioned at $1000 \mathrm{~m}$.

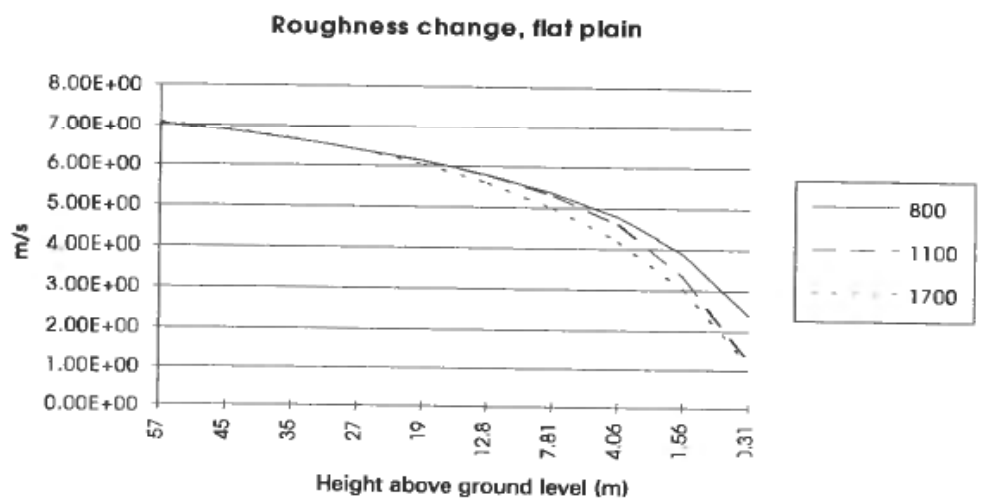

Figure 3. Velocity versus height above ground level $(\mathrm{m})$ for three wind profiles; at $800 \mathrm{~m}$, $1100 \mathrm{~m}$ and $1700 \mathrm{~m}$. The roughness change is located at $1000 \mathrm{~m}$. 


\subsection{Comparison against the Askervein Hill experiments}

Askervein Hill is a smooth hill at the west coast of the Hebrides (Scotland). It has been considered to be a good site for testing wind models.

A series of wind measurements were carried out over Askervein Hill, during 1982-83, see Taylor and Teunissen (1986). Some of the results are reported in Walmsley and Salmon (1984) and compared with the model MS3DJH/3. In Troen and Petersen (1988) the measurements are compared with simulations with WASP(BZ) and MSFD. MSFD is described in Beljaars, Walmsley and Taylor (1986). Both WASP, MSFD and MS3DJH/3 performs well upwind the hill, but at the leeside the codes overestimate the wind speed.

Wind measurements with an upwind direction of $210^{\circ}$ was chosen for the comparison tests. Contour maps of the physical topography of Askervein Hill was obtained by a contouring facility in WASP. A coarse map taken from Troen and Petersen (1988) was magnified and digitized. The contour lines were further processed by the code WGRID to form the coordinates of the lowest grid plane. WGRID creates a uniform grid spacing in the horizontal directions. A separate module was developed to read the WGRID-file, create a vertical grid spacing and write out a 3D grid file in a PHOENICS compatible format.

Figure 4 shows the computational grid together with a contour map of Askervein Hill. The simulated normalised speed (ratio of local speed and upstream speed at same height above ground) at the three cross sections together with the measured values are shown in Figures 5, 6 and 7.

There is good agreement between the simulations and the measurements at all three cross sections. Note the excellent results on the leeside. Upstream the hilltop simulations overpredict the wind speed. At the hilltop, Fig. 6, the wind speed is underpredicted. The main reason for the differences between the measured and the simulated wind speeds are considered to be the coarse contouring and the grid with long and thin cells causing numerical inaccuracy.

\section{Convergence and CPU-time}

\subsection{Convergence}

Initially the simulations were run with inlet conditions (velocity profiles and turbulence parameters) calculated from simplified analytical solutions. These profiles turned out not to be in complete equilibrium with the computed solution, and the convergence was very poor.

The solution to this problem was to let the model itself compute the inlet conditions by simulating wind over a flat plain in parabolic mode. This gave a fully developed boundary layer, which was then used as the inlet conditions for elliptic runs.

\subsection{CPU time}

The simulations were carried out on a workstation (HP 720). Typical computing time for the 3D Askervein Hill run (with grid size $36 \times 58 \times 40$ ) is 75 CPU minutes.

\section{Conclusion}

The Navier-Stokes solver PHOENICS has been used to model microscale atmospheric flow over terrain features. The initial test cases with prescribed density and neutral conditions has demonstrated the models ability to describe influence of 


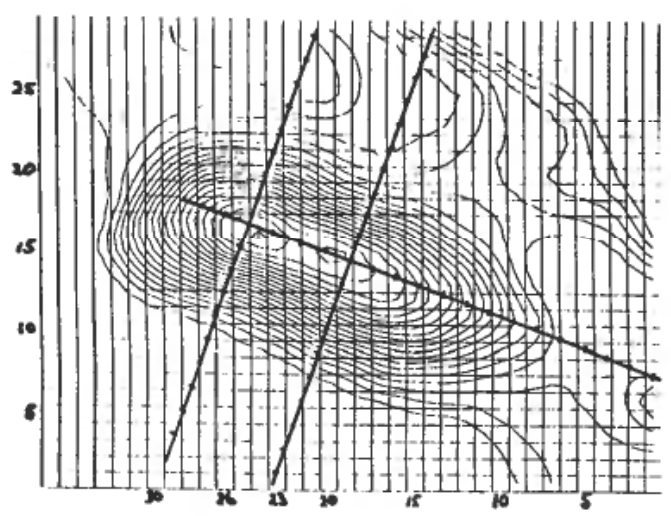

Figure 4. Computational grid and contour map of the 3D Askervein Hill model.

Askervein Hill (ASW-ANE)

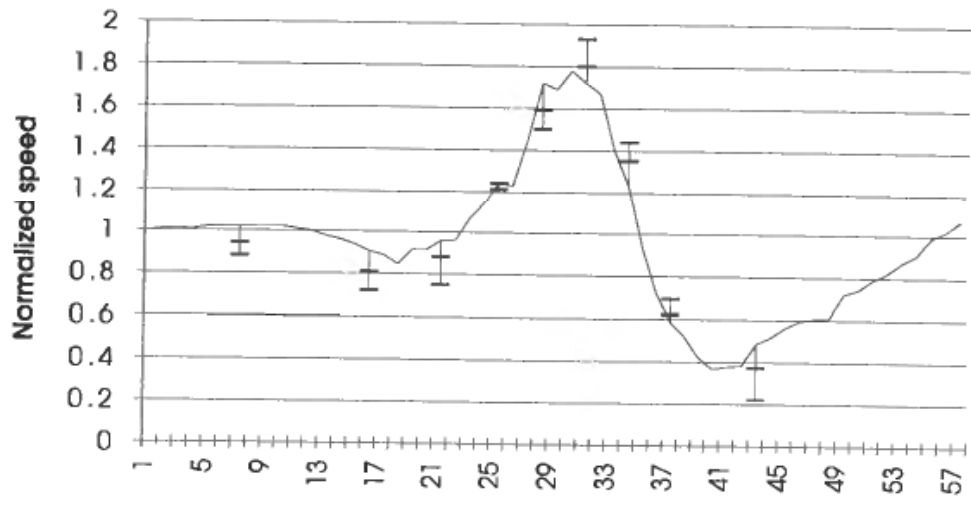

Figure 5. Normalised speed at $10 \mathrm{~m}$ above ground level (a.g.1.) at the SW-NE measurement line through the hilltop (HT).

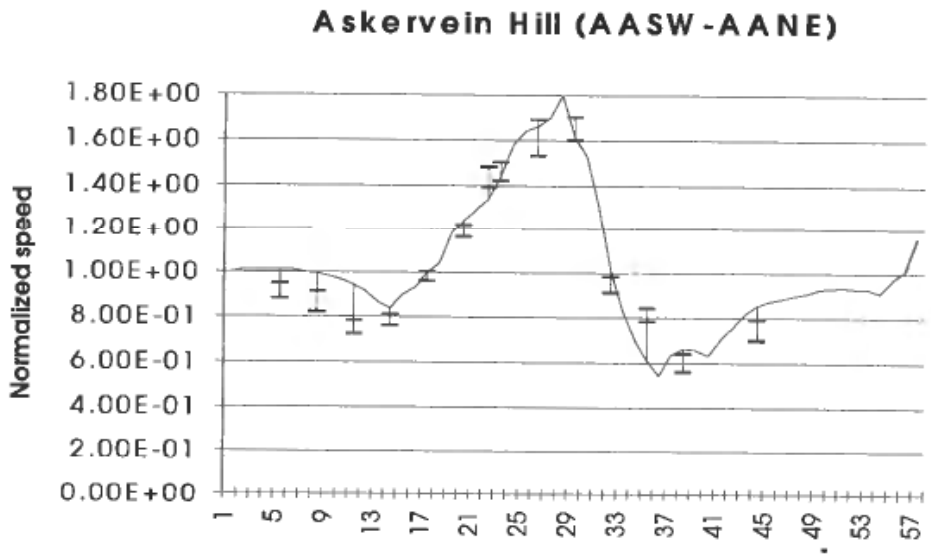

Figure 6. Normalised speed at $10 \mathrm{~m}$ a.g.l. at the SW-NE measurement line through the central point (CP). 
Askervein Hill (BNW-BSE)

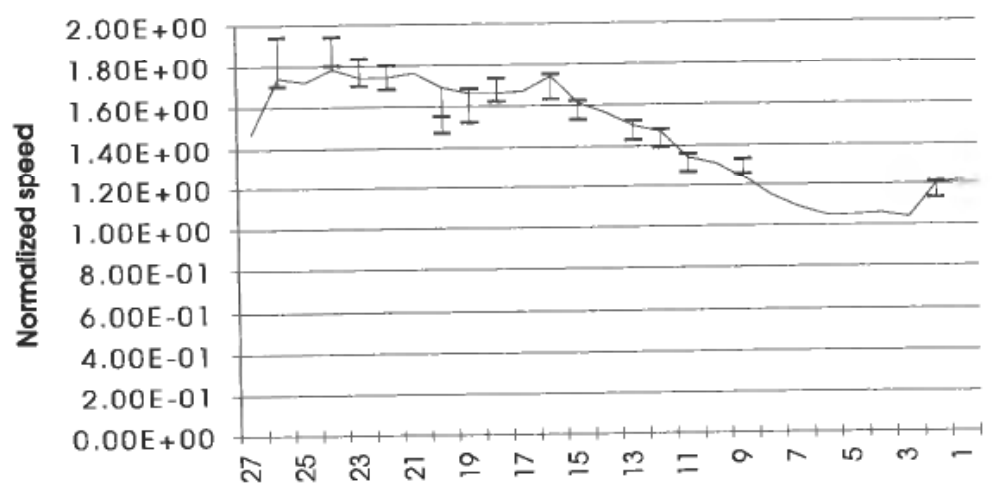

Figure 7. Normalised speed at $10 \mathrm{~m}$ a.g.l. at the NW-SE measurement line at the hill ridge.

roughness change and terrain elevation. The main advantage over simpler linearized models seems to be a better description of the flow behind the hill, where nonlinear effects will be important. When areas with multiple hills are considered, this could give a better representation of the flow field.

\section{ACKNOWLEDGMENTS}

This work has been supported by Department of Trade and Energy through the Norwegian wind energy programme.

\section{REFERENCES}

ANDERSSON, I. (1988). Introduction to turbulence modelling. Lecture notes in Turbulent Flow (NTH, Trondheim).

BELJAARS, A.C.M., WALMSLEY,J.L. and TAYLOR,P.A. (1987). A mixed spectral finite-difference model for neutrally stratified boundary-layer flow over roughness changes and topography. Boundary Layer Meteorology, 38, 273-303.

NYGAARD, T. A. (1992). Estimating energy capture at potential wind turbine sites in Norway, Journal of Wind Engineering and Industrial Aerodynamics, 39, 385-393 (Elsevier Science Publishers B. V., Amsterdam).

PANOFSKY, H. A. and DUTTON, J. A. (1984). Atmospheric Turbulence: Models and Methods for Engineering Applications (John Wiley and Sons, New York).

PATANKAR, S. V. (1980) Numerical heat transfer and fluid flow (Hemisphere Publishing Corporation, New York).

SPALDING, D. B. (1991) The PHOENICS beginner's guide/TR100 (Concentration, Heat and Momentum Limited, London).

TAylor, P. A. and Teunissen, H. W. (1987). The Askervein Hill Project; overview and background data. Boundary Layer Meteorology, 39, 15-39.

Troen, I. and Lundtang, Petersen, E. (1988). Siting of wind turbines; EC Wind Energy Conference, Herning, Denmark (Published by Stephens and Associates, Bedford, England).

WALMSLEY, J. L. and SALMON, J. R. (1984). A boundary layer model for wind flow over hills. Comparison of model results with Askervein 1983 data. European Wind Energy Conference and Exhibition, Hamburg (Published by Stephens and Associates, Bedford, England). 